\title{
Relativistic charged particle in magnetic dipole-spherical geometry III. Local three-dimensional states
}

\author{
Kaundinya S. Gopinath, ${ }^{1}$ Dallas C. Kennedy ${ }^{1}$ and James M. Gelb ${ }^{2}$ \\ ${ }^{1}$ Department of Physics, University of Florida, Gainesville, Florida 32611 USA \\ ${ }^{2}$ Department of Physics, University of Texas at Arlington, Arlington, Texas 76019 USA
}

Accepted ???, Received ???, in original form: UF-IFT-HEP-97-11, July 1997

\begin{abstract}
Following two previous papers, we examine single- and many-body states of relativistic charged particles in an intense, rotating magnetic dipole field. Single-body orbits are derived classically and semiclassically, and then applied to the many-body case via the Thomas-Fermi approximation. Examples of electrons in a realistic neutron star crust are considered with both fixed density profiles and constant Fermi energy. In the first case, the varying magnetic field and Coriolis correction lead to a varying Fermi energy and macroscopic currents; in the second, the electron density is redistributed by the magnetic field. Further questions are outlined.
\end{abstract}

Key words: Neutron stars, pulsars, magnetic fields, elementary processes in plasma

\section{INTRODUCTION}

In two previous papers (Gopinath, Kennedy \& Gelb 1997a, hereafter paper I; Gopinath, Kennedy \& Gelb 1997b, hereafter paper II) we examined the classical and semiclassical relativistic orbits of a particle of mass $m$ and charge $q$ in an intense magnetic field, idealised as a dipole, and a rotating reference frame (papers I \& II). The particle in those cases was confined to a rotating sphere. In this paper, we extend our previous treatment to three-dimensional motion, especially the local cyclotron orbits or Landau states. These results are then applied to many-body electron states defined in a semiclassical (Thomas-Fermi) approximation and calculated in a simplified neutron star crust model with electrons and nuclei.

We ignore gravity (derivatives of the metric) here, as this is negligible for charged particles as compared with the magnetic field. Where numerical values are needed, the tilt angle $\theta_{0}$ between the dipole and rotation axes is assumed to be maximal, $\sin \theta_{0}=1$, and the rotational velocity $\Omega$ to be $\bar{\omega}=\Omega R / c=0.01$, a realistic value for high-field neutron stars with radius $R$ (Shapiro \& Teukolsky 1983; Glendenning 1997).

\section{GENERAL RELATIONS}

(c) 1997 RAS
Figure 1. Geometry of magnetic dipole $\mathbf{M}$ and sphere rotating at angular velocity $\boldsymbol{\Omega}$.

\subsection{Co-ordinates, metric, and field}

As the magnetic dominate rotational effects (unless $r$ is quite large), we place the rotation axis at an angle $\theta_{0}$ with respect to the magnetic dipole in the $\phi=0, \pi$ plane (Fig. 1). The metric in a spherical polar co-ordinate system $(r, \theta, \phi)$ rotating with the sphere is given by the line element

$$
\begin{aligned}
d s^{2}= & g_{\mu \nu} d x^{\mu} d x^{\nu} \\
= & c^{2}\left(1-\omega^{2}\right) d t^{2}-d r^{2}-r^{2}\left(d \theta^{2}+\sin ^{2} \theta d \phi^{2}\right) \\
& -2 c r \omega_{\phi} \sin \theta d t d \phi-2 c r \omega_{\theta} d t d \theta .
\end{aligned}
$$


The vector $\boldsymbol{\omega}$ is defined from the rotational angular velocity vector $\boldsymbol{\Omega}$ by $\boldsymbol{\omega}=\boldsymbol{\Omega} \times \boldsymbol{r} / c$. We also use $\overline{\boldsymbol{\omega}} \equiv \Omega R / c$, where $r$ $=R$ is a reference sphere. The components of $\boldsymbol{\omega}$ are

$$
\begin{aligned}
\omega_{\phi} & =(\bar{\omega} r / R)\left[\cos \theta_{0} \sin \theta-\sin \theta_{0} \cos \theta \cos \phi\right], \\
\omega_{\theta} & =-(\bar{\omega} r / R) \sin \theta_{0} \sin \phi, \\
\omega^{2} & =\omega_{\phi}^{2}+\omega_{\theta}^{2} .
\end{aligned}
$$

These are the appropriate generalisations of papers I \& II and Landau \& Lifshitz (1975) to the case $\sin \theta_{0} \neq 0$ and $r \neq$ constant. The lightsphere is the surface $\boldsymbol{\omega}^{2}=1$, or

$$
\begin{aligned}
\bar{\omega}^{2}(r / R)^{2}\left[\cos ^{2} \theta_{0} \sin ^{2} \theta\right. & +\sin ^{2} \theta_{0} \sin ^{2} \phi \sin ^{2} \theta_{0} \cos ^{2} \theta \cos ^{2} \phi \\
& \left.-(1 / 2) \sin 2 \theta_{0} \sin 2 \theta \cos \phi\right]=1 .
\end{aligned}
$$

This is surface upon which $g_{00}=0$ (Figure 2).

We choose our axes so that the magnetic dipole is along the $\theta=0$ direction. The dipole magnetic field has polar strength $B_{0}$ at $r=R$ :

$$
\begin{aligned}
& A_{\theta}=0 \\
& A_{r}=0 \\
& A_{\phi}=\frac{B_{0} R^{3}}{2 r} \sin ^{2} \theta,
\end{aligned}
$$

where $A_{\phi}$ is covariant in the rotating spherical co-ordinates. For convenience, we rescale $B_{0}$ into dimensionless form as

$\beta_{0}=|q| B_{0} R /\left(2 m c^{2}\right)$.

The magnetic moment of this dipole field is

$|\mathbf{M}|=B_{0} R^{3} / 2$.

In a high-field neutron star, $\beta_{0} \sim 10^{15}$.

The Lagrangian of the charged particle is expressed in terms of the proper time $\tau$, with $\dot{x}^{\mu} \equiv d x^{\mu} / d \tau$. There are four equations of motion, one for each momentum component, but also one energy-momentum constraint. Since the Lagrangian does not explicitly depend on $t$, we have

$\frac{\partial L}{\partial t}=0$

which implies

$\frac{d P_{0}}{d \tau}=0$

Because

$d \tau=\frac{d t \sqrt{g_{\mu \nu}\left(d x^{\mu} / d t\right)\left(d x^{\nu} / d t\right)}}{c}$,

we have also

$\frac{d P_{0}}{d t}=0 \quad$,

where $P_{0}=E$, the energy. The equations of motion for $r, \phi$, and $\theta$ are non-trivial:

$\frac{\partial L}{\partial r}-\frac{d P_{r}}{d \tau}=\frac{\partial L}{\partial \phi}-\frac{d P_{\phi}}{d \tau}=\frac{\partial L}{\partial \theta}-\frac{d P_{\theta}}{d \tau}=0$.

The energy-momentum-mass constraint is

$$
\begin{aligned}
g^{00} P_{0}^{2} & +2 g^{0 i} P_{0}\left(P_{i}-\frac{q}{c} A_{i}\right)+g^{i j}\left(P_{i}-\frac{q}{c} A_{i}\right)\left(P_{j}-\frac{q}{c} A_{j}\right) \\
& =(m c)^{2}
\end{aligned}
$$

Figure 2. Lightsphere defined by (3) in text, surface of $g_{00}=$ 0 , for $\bar{\omega}=0.01$.

including radial and angular terms, but also the mixed rotational $g^{0 \theta}, g^{0 \phi}$ terms. The contravariant metric components are

$$
\begin{array}{cc}
g^{00}=1 / c^{2}, & g^{r r}=-1 / r^{2}, \\
g^{\theta \theta}=-\left(1-\omega_{\theta}^{2}\right) / r^{2}, & g^{\phi \phi}=-\left(1-\omega_{\phi}^{2}\right) /\left(r^{2} \sin ^{2} \theta\right), \\
g^{0 \phi}=-\omega_{\phi} /(c r \sin \theta), & g^{0 \theta}=-\omega_{\theta} /(c r),
\end{array}
$$

Along the actual worldpath in spacetime, the energymomentum constraint is equivalent to $g_{\mu \nu} \dot{x^{\mu}} \dot{x^{\nu}}=c^{2}$. This condition is valid after varying the action and simplifies the equations of motion.

\section{$2.2 \quad$ Asymptotic orbits}

As a check of this dynamical system, we consider briefly the $r \rightarrow \infty$ orbits. The constraint (14) alone is sufficient to give essential information about the trajectory. Note first that $\boldsymbol{\omega}^{2}<1$ for physical trajectories. Since $\boldsymbol{\omega}^{2}=\omega_{\phi}^{2}+\omega_{\theta}^{2}$, each component must also have magnitude less than one. Then $g^{\theta \theta}$ and $g^{\phi \phi}$ are never exactly zero.

In paper I, the natural energy scale for unconfined charged particles at the surface $r=R$ was set by $\epsilon \sim \beta_{0}$, or $E \sim|q| B_{0} R / 2$, independent of $m$ (see also Longair 1992). In the limit $r \rightarrow \infty$, (14) simplifies to

$P_{r}^{2}=(E / c)^{2}-(m c)^{2}$,

where $E$ is naturally $\gtrsim \mathcal{O}\left(|q| B_{0} R\right)$. The magnetic potential term vanishes as $1 / r^{2}$, while the angular momentum terms vanish as $1 / r$. Particles can escape as $r \rightarrow \infty$ if $P_{r}^{2}>0$ and if they remain within the lightsphere surface $\omega^{2}=1$ (Fig. 2). Both graphically and analytically, it is seen that $(\phi, \theta) \rightarrow\left(0, \theta_{0}\right)$ or $\left(\pi, \pi-\theta_{0}\right)$, as $r \rightarrow \infty$. That is, an escaping particle is forced into the common plane of $\mathbf{M}$ and $\boldsymbol{\Omega}$ and leaves along the rotation axis.

For large enough $r$ (strictly speaking, for large enough $|\boldsymbol{\omega}|)$, the magnetic dipole approximation breaks down, and the field (6) can no longer be used. The field lines are twisted about $\Omega$ by the rotation near the lightsphere, and the particle motion is more complex. For example, particles can escape by routes other than along the $\boldsymbol{\Omega}$ axis, depending on the whether the twisted field lines are closed or open (Gol- 
dreich \& Julian 1969). Gravitational attraction is negligible compared to the magnetic force for such energetic particles, even far from the sphere.

\section{APPROXIMATE LOCAL STATES}

The local Landau states can be obtained by expanding the energy-momentum constraint in a Taylor series around $\mathbf{r}=$ $\overline{\mathbf{r}}$, i.e., let $\mathbf{r}$ go to $\overline{\mathbf{r}}+\Delta \mathbf{r}$. The local point is defined by its spherical co-ordinates $(\bar{r}, \bar{\theta}, \bar{\phi})$. We retain terms up to second order in $\boldsymbol{\Delta} \mathbf{r}$ and momentum $\mathbf{P}$, but leave the local metric $g_{\mu \nu}(\overline{\mathbf{r}})$ constant.

\subsection{Local co-ordinates and field}

We define a local Cartesian co-ordinate system so that the $z$ co-ordinate is along the local magnetic field vector, the $x$ co-ordinate is orthogonal to $z$ and pointing outward, and the $y$ co-ordinate is azimuthal.

The dipole magnetic field is

$\mathbf{B}=\left(B_{0} / 2\right)\left(\frac{R}{r}\right)^{3}[2 \cos \theta \hat{\mathbf{r}}+\sin \theta \quad \hat{\boldsymbol{\theta}}]$.

In terms of the local displacement $\Delta \mathbf{r}$, the new co-ordinates are given by

$$
\begin{aligned}
& x=\frac{\Delta r \sin \theta-2 r \Delta \theta \cos \theta}{\sqrt{1+3 \cos ^{2} \theta}}, \\
& z=\frac{2 \Delta r \cos \theta+r \Delta \theta \sin \theta}{\sqrt{1+3 \cos ^{2} \theta}}, \\
& y=-r \sin \theta \Delta \phi
\end{aligned}
$$

while the local Cartesian basis is

$$
\begin{aligned}
& \hat{\mathbf{x}}=\frac{\sin \theta \hat{\mathbf{r}}-2 \cos \theta \hat{\boldsymbol{\theta}}}{\sqrt{1+3 \cos ^{2} \theta}}, \\
& \hat{\mathbf{z}}=\frac{2 \cos \theta \hat{\mathbf{r}}+\sin \theta \hat{\boldsymbol{\theta}}}{\sqrt{1+3 \cos ^{2} \theta}}, \\
& \hat{\mathbf{y}}=-\hat{\boldsymbol{\phi}} .
\end{aligned}
$$

The overline sign of $\overline{\mathbf{r}}$ on each co-ordinate $(r, \theta, \phi)$ is now omitted unless needed.

\subsection{Local Landau states}

The constraint can be rewritten in the local co-ordinates as

$$
\begin{aligned}
\epsilon^{2}- & \Pi_{x}^{2}-\Pi_{z}^{2}-\left(\Pi_{y}^{\prime}-\beta x / r\right)^{2}-1 \\
& \left.+2 \omega_{\phi} \epsilon\left(\Pi_{y}^{\prime}-\beta x / r\right)-2 \omega_{\theta} \epsilon \frac{\sin \theta \Pi_{z}-2 \cos \theta \Pi_{x}}{\sqrt{1+3 \cos ^{2} \theta}}\right)\left[4 \cos \theta x^{2}\right. \\
& +2 \omega_{\phi} \epsilon\left(\frac{\beta \cos \theta}{r^{2} \sin \theta\left(1+3 \cos ^{2} \theta\right)^{3 / 2}}\right) \\
& \left.-3 \cos \theta \sin ^{2} \theta z^{2}+2 \sin \theta\left(3 \cos ^{2} \theta-1\right) x z\right] \\
& +\left(\Pi_{y}^{\prime}-\beta x / r\right)^{2} \omega_{\phi}^{2} \\
& +\frac{\left(\sin \theta \Pi_{z}-2 \cos \theta \Pi_{x}\right)^{2}}{1+3 \cos ^{2} \theta} \omega_{\theta}^{2}=0 .
\end{aligned}
$$

Here we use dimensionless local magnetic field strength, energy and momenta given by

$$
\begin{aligned}
\beta \equiv \frac{|q \mathbf{B}| r}{2 m c^{2}} & =\beta_{0}\left(\frac{R}{r}\right)^{2} \sqrt{1+3 \cos ^{2} \theta}, \\
\epsilon & =E / m c^{2}, \\
\Pi_{x, z} & =P_{x, z} / m c .
\end{aligned}
$$

$\Pi_{y}^{\prime}$ is the new canonical momentum given by the transformation

$$
\Pi_{y}^{\prime}=-\frac{P_{\phi}}{m c r \sin \theta}+\frac{q B_{0} R^{3} \sin \theta}{2 m c^{2} r^{2}} .
$$

The second term is just a shift which is constant in the local co-ordinate system. The first line of the constraint (20) just gives the classical version of the Landau system in terms of the local magnetic field $\mathbf{B}(\overline{\mathbf{r}})$, which also defines the local $z$ axis. The other terms are linear and quadratic corrections (Coriolis and centrifugal terms) due to the local $\boldsymbol{\omega}$ components $\omega_{\phi}$ and $\omega_{\theta}$.

Neglecting rotational effects yields the standard cyclotron motion (Landau \& Lifshitz 1975). In the semiclassical form, these are the Landau orbitals, with energy eigenvalues

$\epsilon_{0}^{2}=1+\Pi_{z}^{2}+\left(\frac{\hbar}{m c}\right)\left(\frac{2 \beta}{r}\right)\left[2 n_{L}+1-\operatorname{sgn}(q) \sigma\right]$,

where the principal Landau quantum number is $n_{L}=0,1$, $2, \ldots$ and the spin $\sigma$ has been included, $\sigma= \pm 1$ (Landau \& Lifshitz 1977; Landau \& Lifshitz 1982). For $\operatorname{sgn}(q)<0$, the state $\left(n_{L}, \sigma=1\right)$ is degenerate with $\left(n_{L}+1, \sigma=-1\right)$. The Landau orbitals are characterised by length and momentum scales $L=\sqrt{\hbar c /(2|q \mathbf{B}|)}$ and $P_{L}=\sqrt{\hbar|q \mathbf{B}| /(2 c)}$ in the $(x, y)$ plane transverse to the field. The longitudinal momentum $P_{z}$ $=m c \Pi_{z}$ is an eigenvalue continuous over the range $-\infty<$ $P_{z}<+\infty$, as the motion in that direction is force-free. Note that the magnetic factor proportional to $\beta$ is suppressed by the ratio of the Compton wavelength $\hbar /(m c)$ to the spherical distance $r$. The effect of the magnetic field in the energy is thus negligible compared to $m c^{2}$ unless $\beta$ is very large, as seen in paper I.

We now consider the effect of rotation as a first-order perturbation in $\bar{\omega}$, approaching the problem semiclassically. (The terms quadratic in $\bar{\omega}$ are much smaller.) The unperturbed orbits are Landau circles in the $(x, y)$ transverse plane. We keep the terms linear in $\boldsymbol{\omega}$ (Coriolis effect) and average them over one Landau orbit, as in paper II. But unlike the that case, this averaging is over a local microscopic orbit, not a macroscopic orbit over the whole sphere. The terms linear in $\bar{\omega}$ thus do not average to zero in general. The modified classical energy-momentum constraint is then

$$
\begin{aligned}
\epsilon^{2}- & \Pi_{x}^{2}-\Pi_{z}^{2}-\left(\Pi_{y}^{\prime}-\beta x / r\right)^{2}-1 \\
& -2 \omega_{\theta} \epsilon \frac{\sin \theta \Pi_{z}}{\sqrt{1+3 \cos ^{2} \theta}} \\
& +2 \omega_{\phi} \epsilon\left(\frac{\beta \cos \theta}{r^{2} \sin \theta\left(1+3 \cos ^{2} \theta\right)^{3 / 2}}\right) \times \\
& {\left[4 \cos \theta x^{2}-3 \cos \theta \sin ^{2} \theta z^{2}\right]=0 . }
\end{aligned}
$$


noting that $\langle x\rangle_{0}=\langle y\rangle_{0}=\left\langle\Pi_{x}\right\rangle_{0}=\left\langle\Pi_{y}\right\rangle_{0}=0$, averaged over an unperturbed cyclotron orbit. These orbits have semiclassical radius $a\left(n_{L}\right)=2 L \sqrt{n_{L}+1 / 2}$. The semiclassical result for the Landau energies corrected through $\mathcal{O}(\bar{\omega})$ is then

$$
\begin{aligned}
\epsilon_{1}^{2}= & \epsilon_{0}^{2}+2 \omega_{\theta} \epsilon_{0} \sin \theta \Pi_{z} / \sqrt{1+3 \cos ^{2} \theta} \\
& -2 \omega_{\phi} \epsilon_{0}\left(\frac{\beta \cos \theta}{r^{2} \sin \theta\left(1+3 \cos ^{2} \theta\right)^{3 / 2}}\right) \times \\
& {\left[4\left(2 n_{L}+1\right) \cos \theta L^{2}-3 \cos \theta \sin ^{2} \theta z_{C}^{2}\right], }
\end{aligned}
$$

where we have used $\left\langle\Pi_{z}\right\rangle_{0}=\Pi_{z},\left\langle x^{2}\right\rangle_{0}=a^{2}\left(n_{L}\right) / 2$, and a distance cutoff $z_{C}$ for motion in the $z$ direction. (This cutoff is discussed further in the next section.) The last term can be neglected if the Landau orbits are much smaller than the spherical distance $r: L, z_{C}<<r$, as it is another factor of $L / r$ smaller than the zeroth-order term. The energies depend on $\Pi_{z}^{2}$ at zeroth order but receive a correction proportional to $\Pi_{z}$ at first order in $\bar{\omega}$, breaking the symmetry $\Pi_{z} \rightarrow-\Pi_{z}$. The Landau pole states of papers I and II can be recovered if $\sin \theta \rightarrow 0, r=R$, identifying $n_{L}$ with the old $n_{\theta}$ and setting $\Pi_{z}=0$.

The error arising from neglecting the cubic terms in the Taylor expansion can be calculated and goes as $\sim|\Delta \mathbf{r}| / r$ times the quadratic terms. That is, the cubic and higher terms in the expansion are suppressed by additional powers of the Landau length $L$ over the sphere size $r$.

\section{MANY-BODY STATES}

\subsection{Density of states}

The full density of states is a product of four factors: the density of Landau states, the degeneracy factor $\mathcal{D}_{\perp}$ in the $(x, y)$ transverse plane for each Landau state, the density of longitudinal states $\mathcal{D}_{\|}$for $z$ motion, and the spin factor (one for the ground state $n_{L}=0$, two otherwise).

The degeneracy of a given Landau state $n_{L}$ in the transverse plane is

$\mathcal{D}_{\perp}=\frac{|q \mathbf{B}|}{2 \pi \hbar c}$

per unit planar area, a result valid in both non-relativistic (Ibach \& Lüth 1995) and relativistic regimes, while the longitudinal motion contributes a factor

$\mathcal{D}_{\|}=\frac{m c d \Pi_{z}}{2 \pi \hbar}$

per unit longitudinal length. Thus the number of states, including the spin factor, is

$$
\frac{d^{2} \mathcal{N}}{d \mathcal{S} d z}=\left(2-\delta_{n_{L}, 0}\right) \cdot \mathcal{D}_{\perp} \cdot \mathcal{D}_{\|}
$$

per unit transverse surface area $d \mathcal{S}$ and unit longitudinal length $d z$.

In the semiclassical limit, where $n_{L}$ is large and quasicontinuous, the density of Landau states per unit energy is given in dimensionless form by

$$
\frac{d n_{L}}{d \epsilon}=\left(\frac{m c r}{4 \beta \hbar}\right)\left[1+\left(2 \omega_{\theta} / \sqrt{1+3 \cos ^{2} \theta}\right)\right]^{-1} \times
$$

Figure 3. Momentum-dependent part $\zeta^{2}$ of the squared energy $\epsilon^{2}$ as a function of longitudinal momentum $\Pi_{z}$, including $\mathcal{O}(\bar{\omega})$ correction, showing the solutions $\Pi_{z}^{ \pm}$of $\zeta^{2}=\zeta_{F}^{2}$.

$$
\begin{aligned}
& {\left[1+\Pi_{z}^{2}+2 \hbar \beta /(m c r)\left[2 n_{L}+1-\operatorname{sgn}(q) \sigma\right]\right.} \\
& +2 \omega_{\theta} \epsilon_{0} \sin \theta\left[\Pi_{z} / \sqrt{1+3 \cos ^{2} \theta}\right]
\end{aligned}
$$

\subsection{Thomas-Fermi approximation}

The Thomas-Fermi method approximates quantum manybody states in a varying potential with local states defined by a locally constant field (Landau \& Lifshitz 1977). In our case, the local states of charged fermions are filled up to some highest and partially-filled Landau level $n_{L}^{*}$ by the charge carriers, assumed here to be electrons $\left(m=m_{e}\right)$. That part $\zeta^{2}$ of the squared energy $\epsilon^{2}$ arising from the $\Pi_{z}$ terms alone:

$\zeta^{2}\left(\Pi_{z}\right)=\Pi_{z}^{2}+2 \omega_{\theta} \epsilon \sin \theta \Pi_{z} / \sqrt{1+3 \cos ^{2} \theta}$

must be cut off at some maximum, as must also the corresponding $z$ motion. In a real system, the cutoffs are provided naturally by the presence of lattice ions: $\left|z_{C}\right| \gtrsim \hbar /\left(Z_{\text {eff }} \alpha m c\right)$ (Bohr length) and $\left|\Pi_{z}\right| \gtrsim Z_{\text {eff }} \alpha$, where $Z_{\text {eff }}$ is an effective (screened) positive ionic charge (Landau \& Lifshitz 1977). In the realistic case, the $z_{C}$ term is unnecessary; assume that $\zeta^{2}<\zeta_{F}^{2}=\left(Z_{\text {eff }} \alpha\right)^{2}$. Then the longitudinal momentum $\Pi_{z}$ is cut off asymmetrically at $\Pi_{z}=\Pi_{z}^{+}>0$ and $\Pi_{z}^{-}<0$, with $\Pi_{z}^{+} \neq-\Pi_{z}^{-}$if $\bar{\omega} \neq 0$ (Fig. 3).

The electron number density $n_{e}$ at any point is given by

$n_{e}=\frac{|q \mathbf{B}|}{2 \pi \hbar c}\left[1+2\left(n_{L}^{*}-1\right)+2 \nu\right] \cdot \Delta \Pi_{z}(m c / 2 \pi \hbar)$,

where $\nu$ is the partial filling factor of the highest Landau level $n_{L}^{*}, 0 \leq \nu \leq 1$, and $n_{L}^{*}$ is assumed $\geq 1$. (If $n_{L}^{*}=0$, the entire term in brackets is replaced by simply $\nu$.) Because of the $z$ degree of freedom, each Landau level is actually a band, with lowest energy level at some $\Pi_{z} \neq 0$, whose sign depends on that of $\omega_{\theta}$. In addition, there is the planar degeneracy, modified by the partial filling factor $\nu$ in the highest Landau level. If $n_{e}$ is specified along with $\Delta \Pi_{z}, n_{L}^{*}$ and $\nu$, and thus $\epsilon_{F}$, can be determined. Further details are found in the Appendix.

If $n_{e}$ is held constant over a sphere of radius $r$, then $n_{L}^{*}, \nu$, and $\epsilon_{F}$ change over that surface as $|\mathbf{B}|$ changes. The 
Figure 4. The last, partially-filled Landau level $n_{L}^{*}$ on spherical surface, at constant density, defined by (31) in text; $B_{0}=10 B_{c}$. (a) $\rho=10 \mathrm{~g} / \mathrm{cm}^{3}$. (b) $\rho=10^{7} \mathrm{~g} / \mathrm{cm}^{3}$. (c) $\rho=10^{13} \mathrm{~g} / \mathrm{cm}^{3}$.

variation of $n_{L}^{*}$ and $\nu$ over the surface is given by

$$
\begin{aligned}
n_{L}^{*}= & \operatorname{Int}\left[\left(\frac{2 \pi \hbar}{m c}\right)^{3}\left(\frac{n_{e}}{\Delta \Pi_{z}}\right)\left(\frac{B_{c}}{B_{0} \sqrt{\alpha}}\right)\left(\frac{r}{R}\right)^{3} \times\right. \\
& \left.\frac{1}{4 \sqrt{1+3 \cos ^{2} \theta}}+\frac{1}{2}\right], \\
\nu= & \left(\frac{2 \pi \hbar}{m c}\right)^{3}\left(\frac{n_{e}}{\Delta \Pi_{z}}\right)\left(\frac{B_{c}}{B_{0} \sqrt{\alpha}}\right) \times \\
& \frac{1}{4 \sqrt{1+3 \cos ^{2} \theta}}+\frac{1}{2}-n_{L}^{*},
\end{aligned}
$$

where $\operatorname{Int}[f]$ in (31) denotes the integer value of the function $f$. Typical variations of $n_{L}^{*}$ and $\nu$ over the sphere are shown in Figs. 4 and 5. The Fermi energy at any point on the surface is

$$
\begin{aligned}
\epsilon_{F}^{2}= & 1+\zeta_{F}^{2}+\left(\frac{B_{0} \sqrt{\alpha}}{B_{c} \pi}\right)\left(\frac{R}{r}\right)^{3}\left(2 n_{L}^{*}+1+\sigma\right) \times \\
& \sqrt{1+3 \cos ^{2} \theta},
\end{aligned}
$$

where $\operatorname{sgn}(q)=-1$, and $\sigma_{F}=-1$ for the $n_{L}^{*}$ level; the critical field strength is $B_{c}=\left(m_{e}^{2} / \pi\right) \sqrt{c^{5} / \hbar^{3}}=1.3 \times 10^{12} \mathrm{G}$, defined in paper I. Matching variations of $\epsilon_{F}$ are shown in Fig. 6. As the Fermi energy $\epsilon_{F}$ varies over a sphere, electron currents flow as the electrons seek the lowest energy; there are also radial currents (see subsection IV.C below). The magnitude and evolution of the currents depend on the electrical resistance. As the magnetic field and rotation affect the electron Fermi energy, the beta equilibria of neutrons and muons are also affected, above their respective thresholds. This last effect cannot be included without revising the hadronic equation of state.

The opposite limit is the case where the Fermi energy is constant in space and no currents flow. Since the field $|\mathbf{B}|$ varies, the electron density $n_{e}$, and thus the positive ion density $n_{p}$, must also. This is the case where the magnetic field is strong enough to dominate the mechanical structure of dense matter. We show three examples of how $n_{e}$ is redistributed over a sphere of constant $r$ for fixed $\epsilon_{F}$ (Fig. 7). The proton density is $n_{p}=\left(Z / Z_{\text {eff }}\right) n_{e}$, and $\rho$ can be inferred from equation (A2).

(C) 1997 RAS, MNRAS 000, 且??
Figure 5. The partial filling factor $\nu$ of the last Landau level $n_{L}^{*}$ on spherical surface at constant density, defined by (31) in text; $B_{0}=10 B_{c}$. (a) $\rho=10 \mathrm{~g} / \mathrm{cm}^{3}$. (b) $\rho=10^{7} \mathrm{~g} / \mathrm{cm}^{3}$. (c) $\rho=10^{13}$ $\mathrm{g} / \mathrm{cm}^{3}$.

Figure 6. The dimensionless Fermi energy $\epsilon_{F}$ on spherical surface at constant density, defined by (32) in text; $B_{0}=10 B_{c}$. (a) $\rho=10 \mathrm{~g} / \mathrm{cm}^{3}$. (b) $\rho=10^{7} \mathrm{~g} / \mathrm{cm}^{3}$. (c) $\rho=10^{13} \mathrm{~g} / \mathrm{cm}^{3}$.

\subsection{Radial structure}

For a simple neutron star, we can assume a given profile of positive ions and thus electrons with radius $r$. With the field $|\mathbf{B}(\mathbf{r})|$ in addition, the radial dependence of $\epsilon_{F}$ can be found. In Fig. 8 the radial profile is shown for $\epsilon_{F}$ from $r / R=$

Figure 7. The electron number density $n_{e}$ on spherical surface at constant Fermi energy, defined by (30) in text; $B_{0}=B_{c}$. Units are inverse Compton volume. (a) $\epsilon_{F}=1.06$. (b) $\epsilon_{F}=5$.0. (c) $\epsilon_{F}$ $=1800$. 
Figure 8. Dimensionless Fermi energy $\epsilon_{F}$ as function of $r / R$ for simplified neutron star crust model of text; $\sin \theta=\sin \phi=1$, and $B_{0}=10 B_{c}$. (a) $r / R=0.7-1.00$. (b) $r / R=0.99-1.00$.
Figure 9. Dimensionless Fermi energy $\epsilon_{F}$ as a function of $r / R$ and $\theta$ for simplified neutron star crust model of text. (a) $\sin \phi=$ $1, r / R=0.7-1.00$, and $B_{0}=10 B_{c} ; \sin \theta>0$. (b) Detail showing $\sin \theta>0.2$.

0.7 to 1.0 , with an expanded subfigure of $r / R=0.99-1.0$. The latter shows the thin surface layer of non-relativistic electrons which changes almost immediately to relativistic electrons as $r$ decreases, with two different limiting slopes. Fig. 9 shows $\epsilon_{F}$ as a function of $r / R$ and $\theta$. The rotational correction drops out in certain cases. If $\sin \phi=0(\overline{\mathbf{r}}$ in the $\mathbf{M}-\boldsymbol{\Omega}$ plane) or $\sin \theta_{0}=0$ (no tilt), $\omega_{\theta}$ vanishes. Also, if $\sin \theta=0$ ( $\overline{\mathbf{r}}$ along the dipole axis), $P_{\theta} \sim r \sin \theta \cdot P_{z} \rightarrow 0$, and the Coriolis effect disappears. Otherwise the $\epsilon_{F}$ radial profile is affected dramatically by the rotational correction for relativistic $\epsilon_{F}$.

For this crust profile, we have used a neutron star model of Glendenning (Glendenning 1997), with only electrons, nuclei, and neutrons, and no muons or other hadrons. The radius and mass of the neutron star are $R=11 \mathrm{~km}$ and $M=$ $1.55 M_{\odot}$, respectively. The profile starts at $r / R=0.7$, where $\rho=2 \times 10^{13} \mathrm{~g} / \mathrm{cm}^{3}$ and decreases outward. As $\epsilon_{F}$ varies in space, currents flow in response. Again, the neutron and muon beta equilibria are changed from their zero-field analogues, but this effect is not treated here.

Now consider the opposite limit again, a constant $\epsilon_{F}$, with $n_{e}(r)$ determined by a given field profile $\mathbf{B}(\mathbf{r}) . \nu$ must be set independently; here we take $\nu=0.5$. The results
Figure 10. The electron number density $n_{e}$ as a function of $r / R$ for constant $\epsilon_{F}=1.06 ; \sin \theta=\sin \phi=1$, and $B_{0}=B_{c}$. Density profile of simplified neutron star crust model in text is too large to show on this scale. Units are inverse Compton volume.

depend sensitively on $\nu$ and $\sigma_{F}$, and require more detailed treatment in combination with gravity to give a realistic picture of the crust structure. Fig. 10 shows as illustrative the $n_{e}$ profile in such a case; for the same surface value of $\epsilon_{F}$, the $n_{e}$ density is too large to be shown on the scale of Fig. 10.

\section{CONCLUSION}

This concludes the classical and semiclassical treatment begun in papers I and II. In this paper, we have found the local one- and many-body states of relativistic charged particles in an intense, rotating magnetic dipole field and spherical geometry.

There remain full quantisation with the Dirac equation and the inclusion of the positively-charged lattice structure. Depending on the degree of lattice order, various conducting or insulating many-body states can arise, affecting the macroscopic currents inferred in section IV. We hope to return to these issues in future publications.

The

independent but related works of Chakrabarty, Bandyopadhyay \& Pal (1997) on nuclear matter in intense magnetic fields and of Canuto (1975) have recently come to our attention.

\section{ACKNOWLEDGMENTS}

We thank Pradeep Kumar of the University of Florida and the U.S. National Science Foundation for his original suggestion and helpful discussions. This work was supported by the Institute for Fundamental Theory and the U.S. Department of Energy Contract No. DE-FG05-86-ER40272 (Univ. Florida).

\section{REFERENCES}

Baym G., Bethe H.A., Pethick C., 1971, Nucl. Phys., A175, 225 Baym G., Pethick C., Sutherland P., 1971, ApJ, 170, 299 
Berestetskii V.B., Lifshitz E.M., Pitaevskii L.P., 1982, Quantum Electrodynamics. Pergamon Press, Oxford

Börner G., 1973, On the Properties of Matter in Neutron Stars in Springer Tracts in Modern Physics vol. 69. Springer-Verlag, Berlin

Canuto V., 1975, Proc. 65th Enrico Fermi School: Physics and Astrophysics of Neutron Stars and Black Holes. North-Holland, Amsterdam

Chakrabarty S., Bandyopadhyay D., Pal S., 1997, Phys. Rev. Lett., 78, 2898

Glendenning N.K., 1997,, Compact Stars: Nuclear Physics, Particle Physics, and General Relativity, Springer-Verlag, New York

Goldreich P., Julian W.H., 1969, ApJ, 157, 839

Gopinath K.S., Kennedy D.C., Gelb J.M., 1997a, University of Florida preprint UF-IFT-HEP-97-2, Los Alamos preprint archive astro-ph \#9702014 (paper I)

Gopinath K.S., Kennedy D.C., Gelb J.M., 1997b, University of Florida preprint UF-IFT-HEP-97-6, Los Alamos preprint archive astro-ph \#9703108 (paper II)

Ibach H., Lüth H., 1995, Solid-State Physics: An Introduction to Principles of Materials Science. Springer-Verlag, New York

Landau, L.D., Lifshitz, E.M., 1975, The Classical Theory of Fields. Butterworth-Heinemann, Oxford

Landau L.D., Lifshitz E.M., 1977, Quantum Mechanics: NonRelativistic Theory. Pergamon Press, Oxford

Longair M.S., 1992, High Energy Astrophysics. Cambridge University Press, Cambridge

Pauling L., 1970, General Chemistry. Dover Publications, New York

Shapiro S.L., Teukolsky S.A., 1983, Black Holes, White Dwarfs, and Neutron Stars: The Physics of Compact Objects. John Wiley \& Sons, New York

\section{APPENDIX A: MANY-BODY THEORY}

To determine the filling of momentum states labelled by $\Pi_{z}$, the cutoff equation $\zeta^{2}\left(\Pi_{z}\right)=\zeta_{F}^{2}$ must be solved for some given value of $\zeta_{F}^{2}$, taken here as $\left(Z_{\text {eff }} \alpha\right)^{2}$. At zeroth order in $\bar{\omega}$, this equation is trivial. Once the rotational corrections are included, the equation is not only quadratic and linear in $\Pi_{z}$, but has a implicit dependence on $\Pi_{z}$ through $\epsilon_{0}$.

In the $\mathcal{O}(\bar{\omega})$ correction, our procedure is to take $\epsilon_{0}^{2}=$ $1+\epsilon_{L}^{2}$, where $\epsilon_{L}^{2}$ is the purely two-dimensional Landau term:

$\epsilon_{L}^{2}=\frac{\hbar|q \mathbf{B}|}{m^{2} c^{3}}\left[2 n_{L}+1-\operatorname{sgn}(q) \sigma\right]$

and neglect the $\Pi_{z}^{2}$ term, as the latter is typically much smaller than one. In that case, the cutoff equation $\zeta^{2}\left(\Pi_{z}\right)=$ $\zeta_{F}^{2}$ is a simple quadratic with the two roots $\Pi_{z}^{ \pm}$and

$$
\begin{aligned}
\Delta \Pi_{z} & =\Pi_{z}^{+}-\Pi_{z}^{-}= \\
& 2 \sqrt{\zeta_{F}^{2}+\omega_{\theta}^{2}\left(1+\epsilon_{L}^{2}\right) \sin ^{2} \theta /\left(1+3 \cos ^{2} \theta\right)}
\end{aligned}
$$

as the allowed spread of $z$ momenta. Given a value of $n_{e}$, the values of $\epsilon_{F}, n_{L}^{*}$, and $\nu$ are found iteratively, starting with $\bar{\omega}=0$, then with this solution used in the $\mathcal{O}(\bar{\omega})$ corrections.

In the opposite case, of fixed $\epsilon_{F}, n_{L}^{*}$ is determined, while we set $\nu=0.5$ for simplicity. The density $n_{e}$ is then determined. For $\sigma_{F}=-1, n_{e}$ is automatically constant with $r$ if $\nu$ $=0.5$, apart from a slight rotational correction (not visible in Figure 10). For any other values of $\nu$ and $\sigma_{F}, n_{e}$ generally increases with $r$. A complete treatment of the bulk crust requires inclusion of nuclear matter and gravity (e.g., see Chakrabarty, Bandyopadhyay \& Pal 1997), as well as an interior $\mathbf{B}$ field profile, not necessarily a dipole.

A semi-realistic spatial profile of electron density requires the proton density $n_{p}=n_{e}$, usually determined in terms of mass density $\rho$. The "effective" electron density $n_{e}$, the density available for conduction, is

$(2 \pi \hbar / m c)^{3} n_{e, \text { cond }}=\frac{Z_{\text {eff }}}{A} \cdot \frac{1.1 \times 10^{-5} \rho}{\mathrm{g} \mathrm{cm}^{-3}}$,

where $Z_{\text {eff }}$ is the number of electrons per nucleus available for conduction (loosely bound electrons). The atomic number $A=N+Z$, where the neutron number $N$ per nucleus is abnormally large for nuclei in an electron Fermi sea. The number density is normalised to a Compton volume $(2 \pi \hbar / m c)^{3}$. From $\rho=10$ to about $3 \times 10^{4} \mathrm{~g} / \mathrm{cm}^{3}$, the inner electrons of the atoms remain bound, not participating in conduction; in this case, $Z_{\text {eff }}<Z$ and can be read off from standard atomic structure (Pauling 1970). The nuclei are always iron $\left(Z=26\right.$ and $\left.Z_{\text {eff }}=8,16,24\right)$ at these densities (Glendenning 1997; Börner 1973). For higher densities, the orbitals of different atoms merge, and $Z_{\text {eff }}=Z$. (We neglect the formation of partially ionised atoms at ultrahigh densities, assuming that all electrons are stripped from their nuclei.) The results do not depend sensitively on $Z_{\text {eff }}$.

For nuclear matter composition at densities below complete nuclear dissociation but for $Z>26$, we use here the results of Baym, Pethick \& Sutherland (1971) and Baym, Bethe \& Pethick (1971) (see also Börner 1973) at $\rho<$ $2 \times 10^{13} \mathrm{~g} / \mathrm{cm}^{3}$. Our method can be applied at higher densities with free nucleons, but needs to incorporate the presence of muons and then of heavier strange and non-strange hadrons, and then possibly of a quark-gluon plasma (Glendenning 1997). In this paper, only the simplest case of electrons and positive ions is examined. 
This figure "Fig97-11.01.gif" is available in "gif" format from: http://arxiv.org/ps/astro-ph/9707196v1 
This figure "Fig97-11.02.gif" is available in "gif" format from: http://arxiv.org/ps/astro-ph/9707196v1 
This figure "Fig97-11.03.gif" is available in "gif" format from: http://arxiv.org/ps/astro-ph/9707196v1 
This figure "Fig97-11.04a.gif" is available in "gif" format from: http://arxiv.org/ps/astro-ph/9707196v1 
This figure "Fig97-11.04b.gif" is available in "gif" format from: http://arxiv.org/ps/astro-ph/9707196v1 
This figure "Fig97-11.04c.gif" is available in "gif" format from: http://arxiv.org/ps/astro-ph/9707196v1 
This figure "Fig97-11.05a.gif" is available in "gif" format from: http://arxiv.org/ps/astro-ph/9707196v1 
This figure "Fig97-11.05b.gif" is available in "gif" format from: http://arxiv.org/ps/astro-ph/9707196v1 
This figure "Fig97-11.05c.gif" is available in "gif" format from: http://arxiv.org/ps/astro-ph/9707196v1 
This figure "Fig97-11.06a.gif" is available in "gif" format from: http://arxiv.org/ps/astro-ph/9707196v1 
This figure "Fig97-11.06b.gif" is available in "gif" format from: http://arxiv.org/ps/astro-ph/9707196v1 
This figure "Fig97-11.06c.gif" is available in "gif" format from: http://arxiv.org/ps/astro-ph/9707196v1 
This figure "Fig97-11.07a.gif" is available in "gif" format from: http://arxiv.org/ps/astro-ph/9707196v1 
This figure "Fig97-11.07b.gif" is available in "gif" format from: http://arxiv.org/ps/astro-ph/9707196v1 
This figure "Fig97-11.07c.gif" is available in "gif" format from: http://arxiv.org/ps/astro-ph/9707196v1 
This figure "Fig97-11.08a.gif" is available in "gif" format from: http://arxiv.org/ps/astro-ph/9707196v1 
This figure "Fig97-11.08b.gif" is available in "gif" format from: http://arxiv.org/ps/astro-ph/9707196v1 
This figure "Fig97-11.09a.gif" is available in "gif" format from: http://arxiv.org/ps/astro-ph/9707196v1 
This figure "Fig97-11.09b.gif" is available in "gif" format from: http://arxiv.org/ps/astro-ph/9707196v1 
This figure "Fig97-11.10.gif" is available in "gif" format from: http://arxiv.org/ps/astro-ph/9707196v1 